\title{
EDITORIAL
}

\section{Editorial for Biomin-10}

\author{
Fu-zhai CUI ${ }^{1}$, Lun BAI ${ }^{2}$ \\ 1 Tsinghua University, Beijing, China \\ 2 Soochow University, Suzhou, China
}

(C) Higher Education Press and Springer-Verlag 2009

In the 10th International Symposium on Biomineralization held in Lianyungang, China, participants from more than 12 countries attended and exchanged the up-to-date understanding of the mechanisms of biomineralization and new strategies for applications of biomineralization principles. About 120 papers were presented in this symposium, part of which including review papers were published in the present issue of Frontiers of Materials Science in China. Part of the papers on biomaterials will be published in an issue of the journal, Biomedical Materials, soon.

Biomineralization as a scientific discipline has experienced an exceptional development and is currently an interdisciplinary field of scientific study, including materials science, chemistry, biology, medicine, engineering sciences, geology, and archaeology. Its potential application is in areas as diverse as medicine, veterinary science, agriculture, astrobiology, environmental engineering, biotechnology, biomimetics and bio-inspired materials science, nanobiotechnology, and so on. In the present proceedings current and important frontiers of biomineralization can be found.

Although the scientific output from the growing field of Biomineralization can be found in numerous and sometimes unrelated journals, the Series of International Symposia on Biomineralization has gathered the most enthusiastic scientists and students from different fields to exchange experimental techniques, reconcile disciplinary jargon, and share concepts, and thereby contribute to increased friendship and collaborative interactions among the Symposium participants.

Previous International Symposia on Biomineralization have been held in:

1 Mainz, Germany, 1970

2 Georgetown, SC, USA, 1974

3 Kashokijima, Japan, 1977

4 Amsterdam, Holland, 1982

5 Arlington, TX, USA, 1986

6 Odawara, Japan, 1990

7 Monaco City, Monaco, 1993

8 Niigata, Japan, 2001

9 Pucon, Chile, 2005

The next symposium is scheduled to be held in Australia in 2012.

\section{Guest Editors}

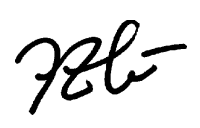

Fu-zhai CUI

And

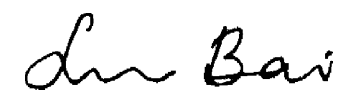

Lun BAI 